\section{Générer une symétrie bilatérale à partir de composants asymétriques}

Cyril Basquin, Noémie Gaudin, Juliette Azimzadeh
Université de Paris, CNRS, Institut Jacques Monod, 15 rue Hélène Brion, 75205 Paris Cedex 13, France. juliette.azimzadeh@ijm.fr
> La grande majorité des animaux présentent une symétrie bilatérale, c'està-dire un plan corporel dans lequel les côtés gauche et droit forment des images en miroir par rapport au plan médian'. Ce schéma d'organisation résulte de la mise en place des axes antéro-postérieur $(A P)$, dorso-ventral (DV), et médio-latéral (ML) au cours de l'embryogenèse. Chez certaines espèces, ces axes peuvent aussi être maintenus de façon dynamique chez l'adulte. C'est le cas des planaires, des vers plats capables de régénérer un organisme entier à partir de pratiquement n'importe quel fragment de leur corps. En réponse à une amputation, les axes corporels peuvent ainsi être rétablis au sein du fragment restant et guider les processus morphogénétiques nécessaires à la régénération d'un animal entier [1].

\section{Une symétrie bilatérale dans \\ l'épiderme de la planaire}

L'épiderme ventral des planaires, qui est entièrement constitué de cellules multiciliées, est un tissu fortement polarisé [1]. Les cellules multiciliées possèdent jusqu'à plusieurs centaines de cils motiles, qui battent de manière coordonnée pour produire un flux de liquide ou déplacer des particules et des cellules [2]. Dans l'espèce humaine, des cellules multiciliées sont notamment impliquées dans la clairance mucociliaire bronchique, un mécanisme qui permet de renouveler la barrière protectrice

${ }^{1}$ Ces animaux bilatériens, ou artiozoaires, sont caractérisés par deux axes de polarité, antéro-postérieur et dorso-ventral. de mucus qui protège les voies respiratoires. Chez les planaires, le battement des cils permet la locomotion. Des animaux dont le corps est rectiligne se déplacent toujours dans le prolongement de l'axe AP (les planaires tournent en contractant leurs muscles d'un côté ou de l'autre du corps), ce qui suggère que le battement ciliaire est lui-même aligné avec l'axe AP, et dirigé vers la partie postérieure. Pour déterminer si tel est le cas, nous avons analysé l'orientation des centrioles - l'organelle cellulaire à partir de laquelle les cils sont assemblés -, dont dépend la direction du battement ciliaire. Les centrioles sont des structures cylindriques qui, dans les cellules multiciliées, portent deux types d'appendices polarisés: le pied basal, qui pointe toujours dans la direction du battement ciliaire, et une racine ciliaire qui pointe dans la direction opposée (Figure 1A) [2, 3]. Pour analyser la polarité rotationnelle des centrioles, c'est-à-dire leur orientation dans le plan de l'épiderme, nous avons utilisé un anticorps marquant la racine ciliaire (Figure 1A). Nous nous sommes ainsi aperçus que chez la planaire, le battement ciliaire est globalement dirigé vers la partie postérieure, mais est aussi en partie dévié vers les bords latéraux, d'autant plus que les cils sont proches du bord [4, 5]. L'ensemble des cils forment ainsi un motif géométrique symétrique par rapport à la ligne médiane (Figure 1C). La symétrie bilatérale de ce motif explique pourquoi le déplacement de l'animal est tout de même aligné avec l'axe AP: la propulsion latérale produite par les cils battant vers l'extérieur est en effet compensée pas celle des cils situés du côté opposé.

Le schéma d'organisation que nous avons mis en évidence révèle que, chez la planaire, la polarité du battement ciliaire est contrôlée le long de deux axes différents, les axes AP et ML. Chez les vertébrés, la polarisation du battement ciliaire à la surface des épithéliums multiciliés implique la voie de signalisation Wnt/PCP (planar cell polarity). Cette voie contrôle la polarité planaire, c'est-àdire l'alignement coordonné de la polarité cellulaire dans le plan d'un tissu $[2,3,6]$. La voie Wnt/PCP implique un ensemble de gènes, identifiés à l'origine chez la drosophile, qui sont très conservés dans le règne animal. Les produits de ces gènes s'organisent en deux complexes de signalisation transmembranaires distribués de façon asymétrique au niveau des jonctions cellulaires pour coordonner la polarité entre cellules voisines (Figure 1B) [6]. Comme nous l'avions supposé, l'inactivation de transcrits codant des composants de la voie Wnt/PCP chez la planaire par une technique d'interférence ARN (ARNi) conduit à une modification de la polarité ciliaire. Remarquablement, on n'observe pas une désorientation complète des cils comme chez les vertébrés, mais une réorientation homogène des cils perpendiculairement aux bords $[4,5]$. Ce résultat suggère l'existence d'une seconde voie agissant le long de l'axe ML et orientant les centrioles vers l'extérieur, et ce d'autant plus que l'influence de la voie Wnt/ PCP diminue. Une autre voie de signalisa- 
A

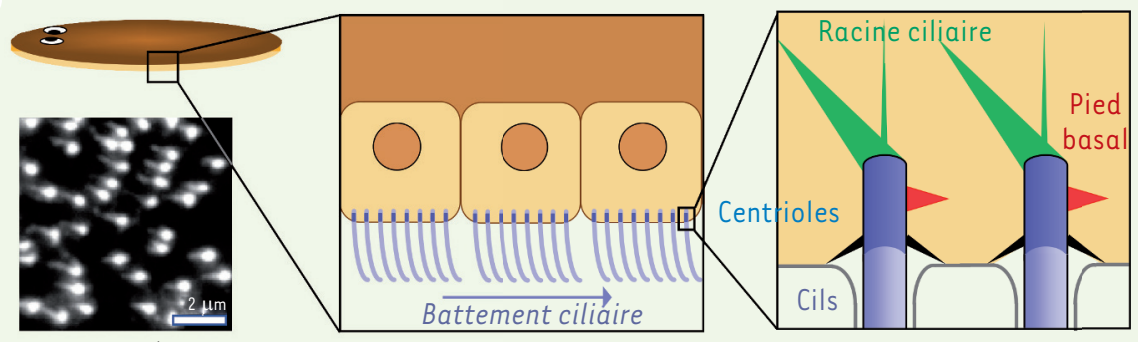

Anti-rootletin

B

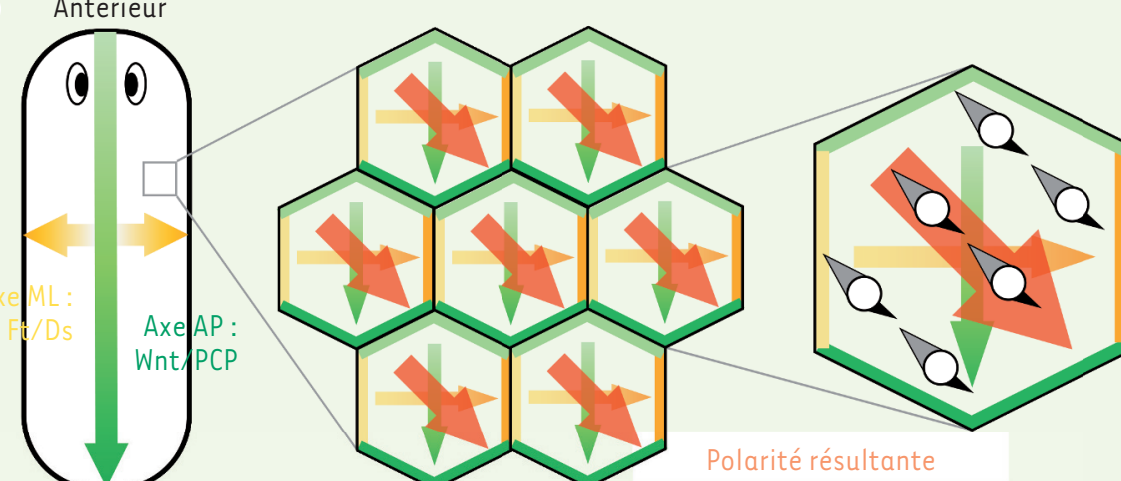

Postérieur

C

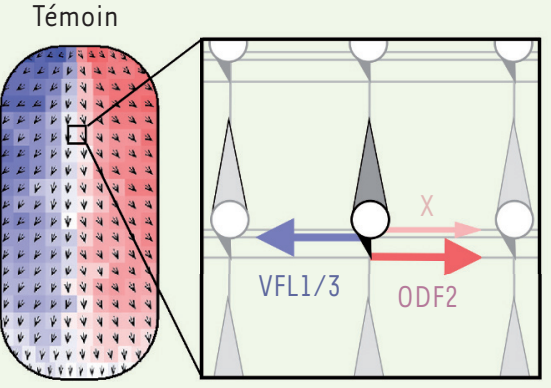

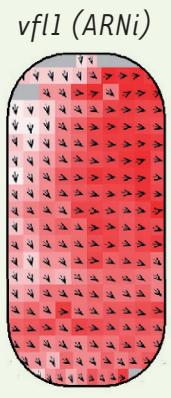

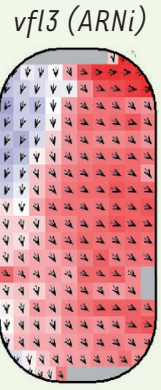

Figure 1. Polarisation de l'épiderme multicilié de la planaire. A. La planaire d'eau douce Schmidtea mediterranea se déplace grâce à un épiderme ventral multicilié (à gauche et au centre). Chaque cil est assemblé à partir d'un centriole doté de deux appendices nécessaires à son positionnement dans le plan de la membrane apicale: le pied basal, qui pointe dans le sens du battement, et la racine ciliaire en orientation opposée (à droite). Il est possible de déduire la polarité du battement ciliaire en marquant la racine par immunofluorescence, grâce à un anticorps dirigé contre son composant principal, la rootlétine (en bas à gauche). B. La polarisation des cils résulte de l'effet conjoint de l'activation de la voie de signalisation Wnt/PCP le long de l'axe antéro-postérieur (AP), et de celle de la voie Ft/Ds le long des deux demi-axes médio-latéraux (ML). Ces deux voies impliquent l'existence d'une distribution asymétrique de complexes protéiques aux jonctions cellulaires (représentés en vert clair et vert foncé ou jaune clair et jaune foncé pour les voies Wnt/PCP

et Ft/Ds, respectivement). Les centrioles s'alignent sur la polarité résultant de l'action simultanée de ces deux voies de signalisation (en rouge). C. La symétrie bilatérale de l'épiderme ventral (à gauche) résulte de l'action des voies de polarité planaire sur un réseau de cytosquelette présentant une asymétrie chirale (au centre). La combinaison des forces exercées par l'intermédiaire de VFL1/3, d'une part, et d'ODF2 et un composant non identifié $(X)$, d'autre part, permet l'alignement des centrioles dans le champ de polarité (au centre). L'asymétrie du réseau de cytosquelette est révélée par une expérience d'inactivation de ces différentes protéines par une technique ARNi (à droite).

tion impliquée dans la polarité planaire est la voie Fat/Dachsous (Ft/Ds, du nom de deux de ses principaux composants moléculaires). Chez la drosophile, où elle est le mieux caractérisée, la voie Ft/ Ds agit dans l'ensemble en orientation parallèle par rapport à la voie Wnt/PCP, à laquelle elle est couplée par l'inter- médiaire de composants moléculaires communs [6]. L'inactivation de la voie $\mathrm{Ft} / \mathrm{Ds}$ chez la planaire entraîne, comme prévu, une perte de la déviation latérale des centrioles, qui sont alors alignés sur I'axe AP, y compris vers les bords. Ainsi, la symétrie bilatérale qui caractérise le plan d'organisation des cils sur la sur- face ventrale de la planaire résulte de l'action combinée des voies Wnt/PCP et $\mathrm{Ft} /$ Ds (Figure 1C). Chez la planaire, ces deux voies de signalisation produisent des champs de polarité orthogonaux sur la plus grande partie du corps de façon permanente, contrairement à ce qui a été décrit chez la drosophile. 
Un réseau de cytosquelette avec une asymétrie chirale

L'organisation des centrioles dans les cellules multiciliées implique la formation de réseaux de cytosquelette qui connectent les centrioles entre eux et aux jonctions intercellulaires, et qui sont polarisés sous l'effet de la signalisation par les voies de la polarité planaire. Des filaments d'actine ancrés aux racines ciliaires et aux centrioles eux-mêmes permettent de contrôler la distribution des centrioles à la surface des cellules et la synchronie du battement ciliaire. Le pied basal, quant à lui, constitue un site d'ancrage pour des microtubules qui contrôlent la polarité rotationnelle des centrioles. Le désassemblage des microtubules ou la perte du pied basal consécutive à l'inactivation du gène codant la protéine 0DF2 (outer dense fiber 2) dans des modèles vertébrés conduit à une orientation aléatoire des centrioles [2, 3]. Chez la planaire, l'inactivation du transcrit codant ODF2 par ARNi bloque aussi la formation du pied basal, mais, étonnamment, l'orientation des centrioles est alors globalement déviée dans le sens horaire en vue de dessus (Figure 1C) [5]. Les animaux déficients en ODF2 se déplacent ainsi en biais vers la droite. D'autre part, lors d'un crible génétique par ARNi, nous avons identifié deux protéines, VFL (variable flagella number) 1 et 3 , pour lesquelles l'inactivation des transcrits correspondants induit le phénotype opposé : les planaires se déplacent en biais vers la gauche. Là encore, l'anomalie de locomotion est corrélée à une déviation des centrioles, cette fois dans le sens antihoraire en vue de dessus (Figure 1C). Par ailleurs, la distribution des centrioles à la surface apicale des cellules est perturbée en l'absence de $V F L 1$ et $V F L 3$, ce qui suggère l'existence d'une désorganisation du cytosquelette d'actine. L'analyse des centrioles par microscopie électronique a révélé différents défauts dans l'assemblage du pied basal et de la racine ciliaire : ces appendices sont souvent absents, et parfois en nombre excessif ou mal positionnés [5]. Ces observations rappellent celles faites à l'origine chez l'algue verte Chlamydomonas reinhardtii, chez laquelle des mutations des gènes vfll et $v f l 3$ entraînent une désorientation des centrioles et des anomalies dans la formation de fibres connectant les centrioles entre eux $[7,8]$. Chez la planaire, VFLl et VFL3 permettent à des éléments du cytosquelette, probablement des filaments d'actine, d'exercer sur les centrioles une force dirigée vers le côté gauche du corps (Figure 1C). Cette force est compensée par d'autres éléments de cytosquelette, dont vraisemblablement des microtubules, ancrés au pied basal et qui exercent une force dirigée vers la droite. L'alignement du battement ciliaire dans ce système résulte donc d'un équilibre des forces qui s'exercent en sens opposés sur les centrioles. Le réseau de cytosquelette organisé autour des centrioles présente ainsi une asymétrie chirale qui, pensons-nous, résulterait d'asymétries structurales des centrioles eux-mêmes.

\section{Conclusion}

La symétrie bilatérale du plan d'organisation des cils motiles de la planaire résulte de l'effet de voies de signalisation de la polarité planaire, activées de façon symétrique de part et d'autre de la ligne médiane, sur un réseau de cytosquelette intrinsèquement asymétrique. Cela implique que la voie Ft/Ds, qui contrôle la polarisation médio-latérale des centrioles et des cils, agit par un mécanisme partiellement différent à gauche et à droite de la ligne médiane pour compenser cette asymétrie. La perturbation concomitante de la polarité planaire et du réseau de centrioles en utilisant des ARNi nous a effectivement permis de mettre en évidence des différences dans la façon dont la voie Ft/ Ds agit sur le réseau de cytosquelette des deux côtés de la ligne médiane. Bien que de nombreuses questions demeurent sans réponse, cette étude permet d'ores et déjà de mieux comprendre comment, chez les animaux bilatériens, un plan d'organisation symétrique peut être mis en place à partir d'éléments constitutifs chiraux. En outre, elle a permis d'identifier de nouveaux acteurs moléculaires nécessaires à la polarisation des épithéliums multiciliés, et qui sont conservés dans l'évolution. Des maladies génétiques affectant la fonction respiratoire dans l'espèce humaine pourraient résulter de la déficience de certains de ces acteurs. $\diamond$

Generating bilateral symmetry from asymmetrical components

\section{LIENS D'INTÉRÊT}

Les auteurs déclarent n'avoir aucun lien d'intérêt concernant les données publiées dans cet article.

\section{RéFÉRENCES}

1. Azimzadeh J, Basquin C. Basal bodies across eukaryotes series: basal bodies in the freshwater planarian Schmidtea mediterranea. Cilia $2016 ; 5: 15$

2. Meunier A, Azimzadeh J. Multiciliated cells in animals. Cold Spring Harbor Perspect Biol 2016 ; 8 : a028233.

3. Brooks ER, Wallingford JB. Multiciliated cells. Curr Biol $2014 ; 24$ : R973-82.

4. Vu HT, Mansour S, Kucken M, et al. Dynamic polarization of the multiciliated planarian epidermis between body plan landmarks. Dev Cell 2019 ; 51 : 526-42 e6.

5. Basquin C, Ershov D, Gaudin N, et al. Emergence of a bilaterally symmetric pattern from chiral components in the planarian epidermis. Dev Cell 2019; $51: 516-25$ e5.

6. Butler MT, Wallingford JB. Planar cell polarity in development and disease. Nat Rev Mol Cell Biol 2017 ; $18: 375-88$.

7. Silflow CD, LaVoie M, Tam LW, et al. The Vfll protein in Chlamydomonas localizes in a rotationally asymmetric pattern at the distal ends of the basal bodies.J Cell Biol 2001 ; 153 : 63-74.

8. Hoops HJ, Wright RL, Jarvik JW, et al. Flagellar waveform and rotational orientation in a Chlamydomonas mutant lacking normal striated fibers. J Cell Biol $1984 ; 98: 818-24$.
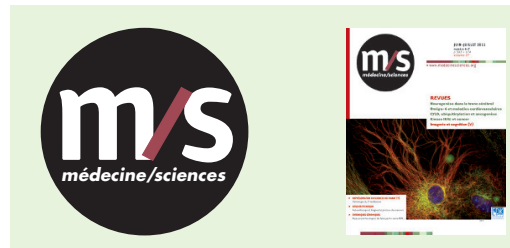

Abonnez-vous

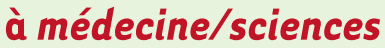

Bulletin d'abonnement page 306 dans ce numéro de $\mathrm{m} / \mathrm{s}$ 